\title{
Cardiovascular Risk Is Elevated in Lean Subjects with Nonalcoholic Fatty Liver Disease
}

\author{
Yuna Kim ${ }^{1,2}$, Eugene Han ${ }^{3}$, Jae Seung Lee ${ }^{1,2,4}$, Hye Won Lee ${ }^{1,2,4}$, Beom Kyung Kim, ${ }^{1,2,4}$, Mi Kyung Kim ${ }^{3}$, Hye Soon \\ $\mathrm{Kim}^{3}$, Jun Yong Park ${ }^{1,2,4}$, Do Young Kim ${ }^{1,2,4}$, Sang Hoon Ahn ${ }^{1,2,4}$, Byung-Wan Lee ${ }^{1,5}$, Eun Seok Kang ${ }^{1,5}$, Bong-Soo \\ Cha ${ }^{1,5}$, Yong-ho Lee ${ }^{1,5}$, and Seung Up Kim ${ }^{1,2,4}$ \\ ${ }^{1}$ Department of Internal Medicine and ${ }^{2}$ Institute of Gastroenterology, Yonsei University College of Medicine, Seoul, ${ }^{3}$ Department \\ of Internal Medicine, Keimyung University School of Medicine, Daegu, ${ }^{4}$ Yonsei Liver Center, Severance Hospital, and ${ }^{5}$ Institute of \\ Endocrine Research, Yonsei University College of Medicine, Seoul, Korea
}

\author{
Article Info \\ Received February 22, 2021 \\ Revised April 28, 2021 \\ Accepted May 12, 2021 \\ Published online July 12, 2021 \\ Corresponding Author \\ Seung Up Kim \\ ORCID https://orcid.org/0000-0002-9658-8050 \\ E-mail ksukorea@yuhs.ac \\ Yong-ho Lee \\ ORCID https://orcid.org/0000-0002-6219-4942 \\ E-mail yholee@yuhs.ac \\ Yuna Kim and Eugene Han contributed \\ equally to this work as first authors.
}

\begin{abstract}
Background/Aims: Nonalcoholic fatty liver disease (NAFLD) and obesity are independently associated with an increased risk for atherosclerotic cardiovascular disease (ASCVD), the leading cause of mortality in patients with NAFLD. Many NAFLD patients are lean, but their ASCVD risk compared to obese subjects with NAFLD is unclear.

Methods: Data from the 2008 to 2011 Korea National Health and Nutrition Examination Surveys database were analyzed $(n=4,786)$. NAFLD was defined as a comprehensive NAFLD score $\geq 40$ or a liver fat score $\geq-0.640$. ASCVD risk was evaluated using the American College of Cardiology/American Heart Association guidelines.

Results: The frequency of subjects without NAFLD, with obese NAFLD, and with lean NAFLD was $62.4 \%(n=2,987), 26.6 \%(n=1,274)$, and $11.0 \% \quad(n=525)$, respectively. Subjects with lean NAFLD had a significantly higher ASCVD score and prevalence of a high ASCVD risk (mean $15.6 \pm 14.0,51.6 \%$ ) than those with obese NAFLD and without NAFLD (mean 11.2 $\pm 11.4,39.8 \%$; mean $7.9 \pm 10.9,25.5 \%$; all $p<0.001$ ). Subjects with lean NAFLD and significant liver fibrosis showed a significantly higher odds ratio for a high risk for ASCVD than those with obese NAFLD with or without significant liver fibrosis (odds ratio, 2.60 vs 1.93; $p=0.023$ ).

Conclusions: Subjects with lean NAFLD had a significantly higher ASCVD score and prevalence of high risk for ASCVD than those with obese NAFLD. Similarly, lean subjects with significant liver fibrosis had a higher probability of ASCVD than obese subjects in the subpopulation with NAFLD. (Gut Liver 2022;16:290-299)
\end{abstract}

Key Words: Nonalcoholic fatty liver disease; Lean; Liver fibrosis; Fatty liver; Cardiovascular risk

\section{INTRODUCTION}

Nonalcoholic fatty liver disease (NAFLD) is the most common liver disease worldwide. Although its prevalence varies geographically, overall it is around $25 \%$ globally. ${ }^{1-3}$ Its increasing prevalence is linked to the alarming increase in obesity, insulin resistance, and diabetes mellitus (DM), which are risk factors for both NAFLD and cardiovascular disease (CVD). Therefore, there is a higher prevalence of CVD among individuals with NAFLD compared to those without NAFLD, and CVD is the leading cause of mortal- ity in individuals with NAFLD. ${ }^{4-7}$

NAFLD is associated with obesity and its related comorbidities, but it can also develop in individuals with a body mass index (BMI) $<25 \mathrm{~kg} / \mathrm{m}^{2}$ in Caucasians and $<23$ $\mathrm{kg} / \mathrm{m}^{2}$ in Asians; these are defined as having lean NAFLD. ${ }^{8}$ This sub-phenotype of patients with NAFLD has been described in various ethnicities, particularly in Asia, and in $10 \%$ to $20 \%$ of nonobese Americans and Caucasians. ${ }^{9,10}$ The pathophysiological mechanisms are unclear and may include dysfunctional adipose tissue, altered body composition, genetic mutations, epigenetic changes early in life, 
and a different gut microbiota composition. ${ }^{10-12}$ Although this phenotype has a more favorable metabolic profile than obese NAFLD, patients with lean NAFLD can develop the full spectrum of liver damage that characterizes non-lean NAFLD. ${ }^{13,14}$ The natural history and long-term prognosis of patients with lean NAFLD are unclear, but lean NAFLD is not a benign condition. Some data suggest it is associated with a worse mortality rate and accelerated disease progression despite a more favorable metabolic risk profile..$^{15,16}$

NAFLD and obesity are independently associated with increased CVD risk. ${ }^{17-19}$ However, the relative risks for patients with obese and lean NAFLD have not been analyzed. Thus, we investigated whether CVD risk differs according to obese versus lean NAFLD and NAFLD with significant liver fibrosis, using data from the Korea National Health and Nutrition Examination Survey (KNHANES).

\section{MATERIALS AND METHODS}

\section{Subjects}

The KNHANES is a nationwide, population-based, cross-sectional health examination and survey that is annually conducted by the Division of Chronic Disease Surveillance of the Korea Disease Control and Prevention Agency of the Ministry of Health and Welfare to monitor the general health and nutritional status of the general civilian population of South Korea. ${ }^{20}$ Subjects are randomly selected from 600 randomly selected districts in cities and provinces of South Korea to provide a representative sam- ple of the Korean population.

As shown in Fig. 1, of the 37,753 subjects in the KNHANES 2008 to 2011 , we initially selected 19,110 subjects aged $\geq 40$ years ( 8,315 men and 10,795 women). Of these, 14,324 were excluded based on insufficient data to calculate the risk for atherosclerotic cardiovascular disease (ASCVD) or a history of ASCVD; positivity for serologic markers of hepatitis B virus or hepatitis C virus, or hepatocellular carcinoma at enrolment or a history thereof; heavy alcohol consumption (>210 g/week for men and $>140 \mathrm{~g} /$ week for women); or insufficient clinical and laboratory information to calculate BMI or the magnitude of liver fibrosis or steatosis.

Written informed consent was secured from all subjects before the study began, and the KNHANES was conducted after approval by the Institutional Review Board of the Korea Disease Control and Prevention Agency (IRB numbers: 2008-04EXP-01-C, 2009-01CON-03-2C, 2010-02CON21-C, and 2011-02CON-06C). The study protocol was also approved by the Institutional Review Board of Yonsei University Health System (IRB number: Y-2020-0133).

\section{NAFLD and liver fibrosis}

NAFLD was defined using previously validated fatty liver prediction models (Supplementary Table 1): the comprehensive NAFLD score (CNS) and the NAFLD liver fat score (LFS); a CNS of $\geq 40$ or an LFS of $\geq-0.640$ were considered indicative of NAFLD. ${ }^{21,22}$ We assessed the fibrotic burden of subjects with NAFLD $(n=1,799)$ using validated liver fibrosis prediction models: the NAFLD fi-

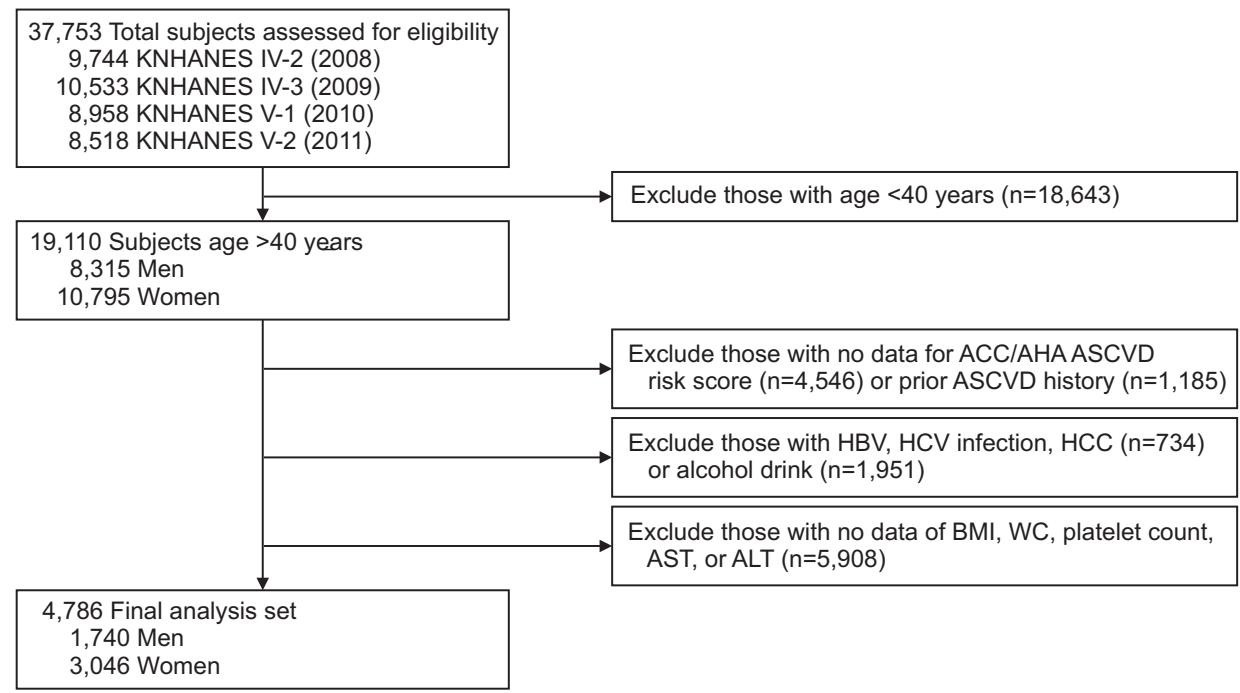

Fig. 1. Flow diagram of subject inclusion and exclusion in the Korea National Health and Nutrition Examination Surveys (KNHANES IV and V). Of 37,753 subjects, 4,786 were ultimately included (1,740 men and 3,046 women).

ACC/AHA, American College of Cardiology/American Heart Association; ASCVD, atherosclerotic cardiovascular disease; HBV, hepatitis B virus; HCV, hepatitis C virus; HCC, hepatocellular carcinoma; BMI, body mass index; WC, waist circumference; AST, aspartate aminotransferase; ALT, alanine aminotransferase. 
brosis score (NFS) and BARD score (Supplementary Table 1) ${ }^{23}$ Significant liver fibrosis was defined as NFS $\geq 0.676$ or BARD score $\geq 2$. Firstly, CNS and NFS were used to define NAFLD and significant liver fibrosis, respectively. Then, LFS and BARD were used for validation.

\section{Assessment of ASCVD risk and cardiometabolic disease components}

ASCVD risk was evaluated using the 10-year ASCVD risk score from the 2013 American College of Cardiology/ American Heart Association (ACC/AHA) guidelines. ${ }^{24}$ Subjects with an ACC/AHA ASCVD risk $>10 \%$ were considered to have a high probability of ASCVD. Based on the criteria for the Asian-Pacific region, ${ }^{25}$ subjects were considered obese at BMI $\geq 25 \mathrm{~kg} / \mathrm{m}^{2}$. Central obesity was defined based on the waist circumference criteria of the Korean Society for the Study of Obesity $(\geq 90 \mathrm{~cm}$ for men and $\geq 85 \mathrm{~cm}$ for women). ${ }^{25} \mathrm{DM}$ was defined based on use of insulin or oral hypoglycemic agents, fasting plasma glucose $\geq 126 \mathrm{mg} / \mathrm{dL}$, or glycated hemoglobin $\geq 6.5 \%$. Subjects were diagnosed as hypertensive at systolic blood pressure $\geq 140 \mathrm{~mm} \mathrm{Hg}$ and diastolic blood pressure $\geq 90 \mathrm{~mm} \mathrm{Hg}$, or if they were taking antihypertensive medications. The estimated glomerular filtration rate was calculated using the epidemiology collaboration equation ${ }^{26}$ and chronic kidney disease (CKD) was defined as estimated glomerular filtration rate $<60 \mathrm{~mL} / \mathrm{min} / 1.73 \mathrm{~m}^{2} .^{27}$ Hyper-low-density lipoprotein (LDL) cholesterolemia was defined according to the 2004 update of the Adult Treatment Panel III guidelines, or current use of an anti-dyslipidemia drug (e.g., statin) for both sexes. ${ }^{28}$ Hypo-high-density lipoprotein (HDL) cholesterolemia was defined as $\mathrm{HDL}<40 \mathrm{mg} / \mathrm{dL}$ for men and $<50 \mathrm{mg} / \mathrm{dL}$ for women. Hypertriglyceridemia was defined as serum levels of triglyceride $\geq 150 \mathrm{mg} / \mathrm{dL}$ or use of triglyceride-lowering agents. Proteinuria was defined as more than one positive dip-stick test or urine albumin-tocreatinine ratio $>30 \mathrm{mg} / \mathrm{g}$, according to the guidelines of the Kidney Disease Outcomes Quality Initiative. ${ }^{27}$ Proteinuria was assessed in 4,588 subjects via urinalysis.

\section{Clinical parameters and biochemical analyses}

The KNHANES data include medical history, smoking habit, alcohol consumption, exercise level, and disease diagnosis and/or treatment, based on direct interviews and self-reporting using standardized health questionnaires. ${ }^{29}$ Smoking status was categorized as non- or current smoker. Regular exercise was defined as engaging in moderate or vigorous exercise on a regular basis ( $\geq 20$ minutes at a time, at least three times per week). ${ }^{30}$

After an overnight fast ( $\geq 8$ hours), blood specimens were collected, processed, and transported in cold storage to the Central Testing Institute (Neodin Medical Institute, Seoul, Korea). Blood samples were analyzed within 24 hours of transportation, as described previously. The homeostasis model assessment of insulin resistance (HOMA-IR) was assessed as reported elsewhere. ${ }^{31}$

\section{Statistical analyses}

The characteristics of the subjects were analyzed according to NAFLD and obesity status using one-way analysis of variance for continuous variables and chi-square tests for categorical variables, followed by post hoc analyses using the Bonferroni method. The association between ASCVD risk and obesity in NAFLD was evaluated using a chisquare test after transforming the variables into quartiles.

Multivariate logistic regression analyses were performed to determine the independent association between high ASCVD risk (ACC/AHA ASCVD risk >10\%) and obesity status in NAFLD after adjusting for age and sex in model 1. The variables in model 1 (smoking, exercise, waist circumference, hypertension, DM, HOMA-IR, CKD, hyper-LDL cholesterolemia, and hypo-HDL cholesterolemia) were adjusted in model 2 . In addition, to assess the effects of a high ASCVD risk, the adjusted model 2 was applied to NAFLD, obesity, hypertension, DM, smoking, exercise, CKD, hyper-LDL cholesterolemia, and hypo-HDL cholesterolemia. Finally, we evaluated whether NAFLD with liver fibrosis was independently associated with a high ASCVD risk in the subpopulation with NAFLD.

Because the total cholesterol, triglyceride, HDL cholesterol, LDL cholesterol, insulin, and HOMA-IR values were not normally distributed, analyses were performed using log-transformed data to achieve approximately symmetrical distributions. Continuous and categorical variables are expressed as means \pm standard deviations and numbers (\%), respectively. A $p<0.05$ was considered indicative of statistical significance. Statistical analyses were performed using Statistical Package for the Social Sciences version 23.0 for Windows (IBM Corp., Armonk, NY, USA).

\section{RESULTS}

\section{Baseline characteristics of the study population}

After excluding 14,324 subjects according to the exclusion criteria, 4,786 subjects (1,740 men and 3,046 women) were included in the statistical analyses (Fig. 1). Baseline characteristics according to NAFLD and obesity status are shown in Table 1. The frequencies of subjects without NAFLD, obese subjects with NAFLD, and lean subjects with NAFLD were $62.4 \%(n=2,987), 26.6 \%(n=1,274)$, and $11.0 \%(\mathrm{n}=525)$, respectively. 
Table 1. Baseline Characteristics

\begin{tabular}{|c|c|c|c|c|}
\hline Variable & $\begin{array}{l}\text { Subjects without NAFLD } \\
(n=2,987,62.4 \%)\end{array}$ & $\begin{array}{l}\text { Obese subjects with NAFLD } \\
\quad(n=1,274,26.6 \%)\end{array}$ & $\begin{array}{l}\text { Lean subjects with NAFLD } \\
\qquad(n=525,11.0 \%)\end{array}$ & p-value \\
\hline \multicolumn{5}{|l|}{ Demographic variables } \\
\hline Age, yr & $56.6 \pm 11.7$ & $58.2 \pm 10.6^{\dagger}$ & $60.5 \pm 10.8^{+, \pm}$ & $<0.001$ \\
\hline Male sex & 924 (30.9) & $513(40.3)^{+}$ & $303(57.7)^{+. \ddagger}$ & $<0.001$ \\
\hline Waist circumference, $\mathrm{cm}$ & $77.4 \pm 7.0$ & $91.9 \pm 6.4^{+}$ & $85.4 \pm 6.1^{1, \neq}$ & $<0.001$ \\
\hline Body mass index, $\mathrm{kg} / \mathrm{m}^{2}$ & $22.3 \pm 2.2$ & $27.5 \pm 2.2^{\dagger}$ & $23.7 \pm 1.1^{+, \neq}$ & $<0.001$ \\
\hline Systolic blood pressure, $\mathrm{mm} \mathrm{Hg}$ & $118.4 \pm 17.6$ & $125.8 \pm 16.4^{+}$ & $124.8 \pm 17.0^{+}$ & $<0.001$ \\
\hline Diastolic blood pressure, $\mathrm{mm} \mathrm{Hg}$ & $73.8 \pm 10.0$ & $78.7 \pm 10.0^{+}$ & $76.6 \pm 10.5^{+, \neq}$ & $<0.001$ \\
\hline Hypertension & $824(27.6)$ & $684(53.7)^{\dagger}$ & $251(47.8)^{+}$ & $<0.001$ \\
\hline Metabolic syndrome & $541(18.1)$ & $942(73.9)^{+}$ & $377(71.8)^{\dagger}$ & $<0.001$ \\
\hline Diabetes mellitus & $153(5.1)$ & $291(22.8)^{\dagger}$ & $171(32.6)^{+, \pm}$ & $<0.001$ \\
\hline Current smoker & 365 (12.2) & $184(14.4)$ & $118(22.5)^{+, \ddagger}$ & $<0.001$ \\
\hline Central obesity & $710(23.8)$ & $1,090(85.6)^{+}$ & $242(11.8)^{+, \ddagger}$ & $<0.001$ \\
\hline Obesity & 337 (11.3) & $1,274(100)^{+}$ & $0^{+, \neq}$ & $<0.001$ \\
\hline Exercise & $472(15.8)$ & $191(15.0)$ & 64 (12.2) & 0.046 \\
\hline \multicolumn{5}{|l|}{ Laboratory variables } \\
\hline Fasting blood glucose, $\mathrm{mg} / \mathrm{dL}$ & $93.8 \pm 12.7$ & $108.3 \pm 28.7^{+}$ & $117.3 \pm 40.6^{\dagger . \neq}$ & $<0.001$ \\
\hline Insulin, $\mu \mathrm{IU} / \mathrm{mL}^{*}$ & $8.6 \pm 3.2$ & $12.6 \pm 5.8^{\dagger}$ & $10.9 \pm 5.8^{+, \neq}$ & $<0.001$ \\
\hline Homeostatic model assessment of insulin resistance* & $2.0 \pm 0.9$ & $3.4 \pm 2.0^{+}$ & $3.1 \pm 2.0^{\dagger . \ddagger}$ & $<0.001$ \\
\hline Total cholesterol, mg/dL & $191.2 \pm 34.5$ & $202.4 \pm 37.5^{+}$ & $199.4 \pm 38.0^{+}$ & $<0.001$ \\
\hline Triglyceride, mg/dL* & $104.3 \pm 54.6$ & $175.2 \pm 95.7^{+}$ & $219.4 \pm 128.4^{+. \ddagger}$ & $<0.001$ \\
\hline High density lipoprotein cholesterol, mg/dL* & $54.5 \pm 12.4$ & $47.0 \pm 10.5^{\dagger}$ & $43.9 \pm 9.6^{+. \neq}$ & $<0.001$ \\
\hline Low density lipoprotein cholesterol, mg/dL* & $119.8 \pm 30.7$ & $125.8 \pm 34.0^{+}$ & $119.0 \pm 34.2^{+}$ & $<0.001$ \\
\hline Serum creatinine, $\mathrm{mg} / \mathrm{dL}$ & $0.8 \pm 0.2$ & $0.9 \pm 0.2^{+}$ & $0.9 \pm 0.2^{\ddagger}$ & $<0.001$ \\
\hline Estimated glomerular filtration rate, $\mathrm{mL} / \mathrm{min} / 1.73 \mathrm{~m}^{2}$ & $89.8 \pm 15.4$ & $86.1 \pm 16.1^{\dagger}$ & $85.1 \pm 14.8^{\ddagger}$ & $<0.001$ \\
\hline Aspartate aminotransferase, IU/L* & $20.7 \pm 6.8$ & $25.0 \pm 11.5^{\dagger}$ & $25.4 \pm 10.8^{+, \neq}$ & $<0.001$ \\
\hline Alanine aminotransferase, IU/L* & $16.7 \pm 7.4$ & $28.0 \pm 18.8^{\dagger}$ & $29.6 \pm 18.2^{\ddagger}$ & $<0.001$ \\
\hline Platelet count, $10^{9} / L^{*}$ & $255.2 \pm 58.7$ & $257.4 \pm 58.9$ & $251.5 \pm 25.5$ & 0.950 \\
\hline Gamma glutamyl-transpeptidase, IU/L* & $24.0 \pm 41.9$ & $39.6 \pm 44.4^{+}$ & $50.6 \pm 69.2^{+, \ddagger}$ & $<0.001$ \\
\hline Proteinuria & $28(1.0)$ & $36(2.9)^{+}$ & $17(3.3)^{\dagger}$ & $<0.001$ \\
\hline \multicolumn{5}{|l|}{ Liver steatosis } \\
\hline Comprehensive NAFLD score & $13.7 \pm 11.5$ & $73.4 \pm 17.2^{\dagger}$ & $59.4 \pm 14.6^{+. \ddagger}$ & $<0.001$ \\
\hline NAFLD liver fat score & $16.1 \pm 12.3$ & $56.1 \pm 19.4^{\dagger}$ & $44.2 \pm 16.9^{+. \ddagger}$ & $<0.001$ \\
\hline
\end{tabular}

Data are presented as mean \pm SD or number $(\%)$.

NAFLD, nonalcoholic fatty liver disease.

*Log-transformed; ${ }^{\dagger} p<0.05$ by post hoc analyses compared without NAFLD; ${ }^{\ddagger} p<0.05$ by post hoc analyses compared with obese NAFLD.

Obese subjects with NAFLD were significantly older (mean 58.2 years vs 56.6 years) and there was a significantly higher proportion of males' sex those without NAFLD ( $40.3 \%$ vs $30.9 \%$ ) (all $\mathrm{p}<0.05$; hereafter, all $\mathrm{p}<0.05$ unless otherwise noted); the same was true among lean subjects (mean 60.5 years vs 56.6 years; $57.7 \%$ vs $30.9 \%$ ). Subjects with NAFLD, regardless of obese or lean status, showed unfavorable characteristics, such as larger waist circumference, higher BMI, higher blood pressure, and higher frequencies of hypertension, metabolic syndrome, DM, central obesity, and obesity compared to those without NAFLD.

Compared to subjects with obese NAFLD, those with lean NAFLD were significantly older (mean 60.5 years vs 58.2 years) and there were significantly more males ( $57.7 \%$ vs $40.3 \%$ ). They had significantly lower waist circumferences (mean $85.4 \mathrm{~cm}$ vs $91.9 \mathrm{~cm}$ ), BMIs (mean $23.7 \mathrm{~kg} / \mathrm{m}^{2}$ vs 27.5 $\mathrm{kg} / \mathrm{m}^{2}$ ), and diastolic blood pressures (mean $76.6 \mathrm{~mm} \mathrm{Hg}$ vs $78.7 \mathrm{~mm} \mathrm{Hg}$ ) but higher rates of diabetes (32.6\% vs $22.8 \%$ ) and current smoking (22.5\% vs $14.4 \%$ ). In addition, they had significantly higher levels of fasting blood glucose (mean $117.3 \mathrm{mg} / \mathrm{dL}$ vs $108.3 \mathrm{mg} / \mathrm{dL}$ ), triglycerides (mean 219.4 $\mathrm{mg} / \mathrm{dL}$ vs $175.2 \mathrm{mg} / \mathrm{dL}$ ), aspartate aminotransferase (mean $25.4 \mathrm{IU} / \mathrm{L}$ vs $25.0 \mathrm{IU} / \mathrm{L}$ ), alanine aminotransferase (mean $29.6 \mathrm{IU} / \mathrm{L}$ vs $28.0 \mathrm{IU} / \mathrm{L}$ ), and gamma glutamyl-transpeptidase (mean $50.6 \mathrm{IU} / \mathrm{L}$ vs $39.6 \mathrm{IU} / \mathrm{L}$ ) levels, but significantly lower levels of insulin (mean $10.9 \mu \mathrm{IU} / \mathrm{mL}$ vs $12.6 \mu \mathrm{IU} /$ mL), HOMA-IR (mean 3.1 vs 3.4), HDL (mean $43.9 \mathrm{mg} / \mathrm{dL}$ vs $47.0 \mathrm{mg} / \mathrm{dL}$ ), LDL (mean $119.0 \mathrm{mg} / \mathrm{dL}$ vs $125.8 \mathrm{mg} / \mathrm{dL}$ ), and estimated glomerular filtration rate (mean $85.1 \mathrm{~mL} /$ $\mathrm{min} / 1.73 \mathrm{~m}^{2}$ vs $86.1 \mathrm{~mL} / \mathrm{min} / 1.73 \mathrm{~m}^{2}$ ). Finally, they had a significantly lower CNS (mean 59.4 vs 73.4) and LFS (mean 44.2 vs 56.1). The frequencies of hypertension, metabolic syndrome, and regular exercise were similar between the 
groups. As expected, CNS and LFS were significantly higher in subjects NAFLD than in those without NAFLD (mean 73.4 and 59.4 vs 13.7 for CNS; mean 56.1 and 44.2 vs 16.1 for LFS).

\section{Association between ASCVD risk and NAFLD/ obesity status}

ASCVD scores and the relative risk of ASCVD according to NAFLD/obesity status are shown in Fig. 2. Subjects with lean NAFLD had a significantly higher ASCVD score and prevalence of a high ASCVD risk (mean 15.6 \pm 14.0 , $51.6 \%$ ), followed by subjects with obese NAFLD and those without NAFLD (mean 11.2 $\pm 11.4,39.8 \%$; mean $7.9 \pm 10.9$, $25.5 \%$; respectively, all $\mathrm{p}<0.001)$. Similar findings were obtained when NAFLD was defined using the LFS (Supplementary Fig. 1). When obesity was defined as BMI $\geq 30$ $\mathrm{kg} / \mathrm{m}^{2}$, ASCVD scores and the proportion of high ASCVD risk among obese NAFLD subjects were significantly lower than those among lean NAFLD subjects (mean 11.2 \pm 11.4 , $39.8 \%$ vs mean $15.6 \pm 14.0,51.6 \%$; all $\mathrm{p}<0.001$ ) (Supplementary Fig. 2).

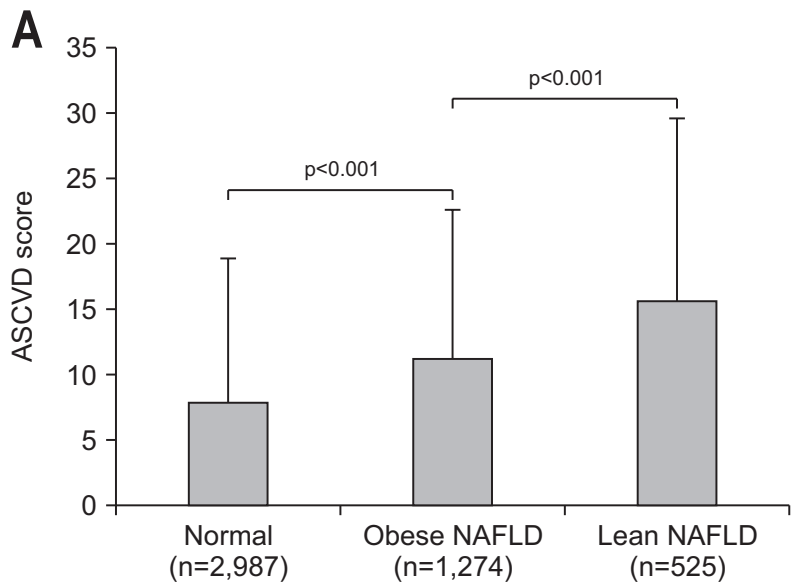

\section{Relative risks for cardiometabolic risk factors}

The adjusted relative risks for cardiometabolic factors were analyzed when NAFLD was defined based on the CNS (Table 2). Risks for hypertension, DM, hypo-HDI cholesterolemia, hypertriglyceridemia, and proteinuria were significantly higher in the lean group whereas the risks of CKD and hyper-LDL cholesterolemia were significantly higher in the obese group. Next, we repeated the analysis after defining NAFLD based on the LFS (Supplementary Table 2)

\section{Association between a high probability of ASCVD and NAFLD/obesity status}

The associations between a high probability of ASCVD and NAFLD/obesity status with multistep adjustments are shown in Table 3. When CNS was used to define NAFLD and the relative risk of ASCVD was assessed after sufficient adjustment (model 2), lean subjects had a higher OR for a high probability of ASCVD than those with obese NAFLD (odds ratio 2.63 vs 2.05 ; all $\mathrm{p}<0.001$ ) or subjects without NAFLD. We also repeated the analysis after defin-

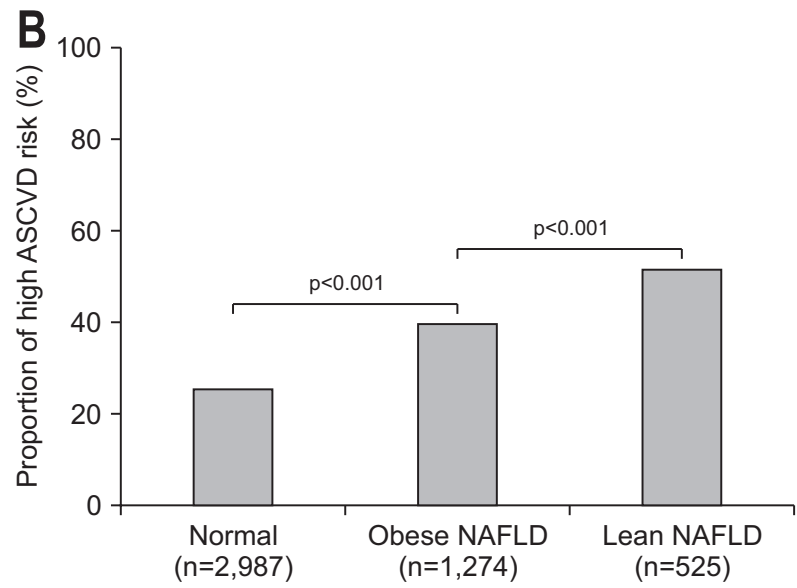

Fig. 2. ASCVD score and proportion of patients with high ASCVD risk according to the CNS-defined NAFLD/obesity status. Subjects with lean NAFLD had a significantly higher ASCVD score (A) and prevalence of a high ASCVD risk (B) than subjects with obese NAFLD and those without NAFLD (all $p<0.001$ ).

ASCVD, atherosclerotic cardiovascular disease; NAFLD, nonalcoholic fatty liver disease; CNS, comprehensive NAFLD score.

Table 2. Cardiometabolic Risk Factors Stratified by Obesity and NAFLD Status Using the Comprehensive NAFLD Score

\begin{tabular}{|c|c|c|c|c|c|}
\hline \multirow{2}{*}{ Variable } & \multirow{2}{*}{$\begin{array}{l}\text { Subjects without NAFLD } \\
\text { OR }(95 \% \mathrm{CI})\end{array}$} & \multicolumn{2}{|c|}{ Obese subjects with NAFLD } & \multicolumn{2}{|c|}{ Lean subjects with NAFLD } \\
\hline & & OR $(95 \% \mathrm{Cl})$ & $p$-value & OR $(95 \% \mathrm{Cl})$ & $\mathrm{p}$-value \\
\hline Hypertension & 1.00 (reference) & $3.23(2.78-3.73)$ & $<0.001$ & $5.40(4.37-6.68)$ & $<0.001$ \\
\hline Diabetes mellitus & 1.00 (reference) & $5.40(4.37-10.29)$ & $<0.001$ & $8.00(6.22-10.29)$ & $<0.001$ \\
\hline Chronic kidney disease & 1.00 (reference) & $1.85(1.37-2.50)$ & $<0.001$ & $1.50(1.01-2.23)$ & 0.043 \\
\hline Hyper-LDL cholesterolemia & 1.00 (reference) & $2.24(1.94-2.59)$ & $<0.001$ & $2.09(1.71-2.55)$ & $<0.001$ \\
\hline Hypo-HDL cholesterolemia & 1.00 (reference) & $2.94(2.55-3.39)$ & $<0.001$ & $4.29(3.48-5.31)$ & $<0.001$ \\
\hline Hypertriglyceridemia & 1.00 (reference) & $5.46(4.72-6.30)$ & $<0.001$ & $10.29(8.29-12.76)$ & $<0.001$ \\
\hline Proteinuria & 1.00 (reference) & $2.90(1.76-4.77)$ & $<0.001$ & $3.01(1.61-5.61)$ & 0.001 \\
\hline
\end{tabular}

Adjusted for age and sex.

NAFLD, nonalcoholic fatty liver disease; OR, odds ratio; Cl, confidence interval; LDL, low-density lipoprotein; HDL, high-density lipoprotein 
Table 3. High Probability of ASCVD According to Obesity and NAFLD Based on the Comprehensive NAFLD Score

\begin{tabular}{|c|c|c|c|c|c|}
\hline \multirow{2}{*}{ Model } & \multirow{2}{*}{$\begin{array}{l}\text { Subjects without } \\
\text { NAFLD OR (95\% CI) }\end{array}$} & \multicolumn{2}{|c|}{ Obese subjects with NAFLD } & \multicolumn{2}{|c|}{ Lean subjects with NAFLD } \\
\hline & & OR $(95 \% \mathrm{CI})$ & $p$-value & OR (95\% CI) & $\mathrm{p}$-value \\
\hline Crude & 1.00 (reference) & $1.93(1.68-2.22)$ & $<0.001$ & $3.12(2.58-3.77)$ & $<0.001$ \\
\hline Model 1 & 1.00 (reference) & $3.68(2.89-4.69)$ & $<0.001$ & $3.71(2.68-5.14)$ & $<0.001$ \\
\hline Model 2 & 1.00 (reference) & $2.05(1.37-3.07)$ & 0.001 & $2.63(1.61-3.58)$ & $<0.001$ \\
\hline
\end{tabular}

ASCVD, atherosclerotic cardiovascular disease; NAFLD, nonalcoholic fatty liver disease; OR, odds ratio; $\mathrm{Cl}$, confidence interval.

Model 1: adjusted for age and sex and model 2: adjusted for age, sex, smoking, exercise, waist circumference, hypertension, diabetes, homeostasis model assessment of insulin resistance, chronic kidney disease, and hyper-low-density lipoprotein cholesterolemia.
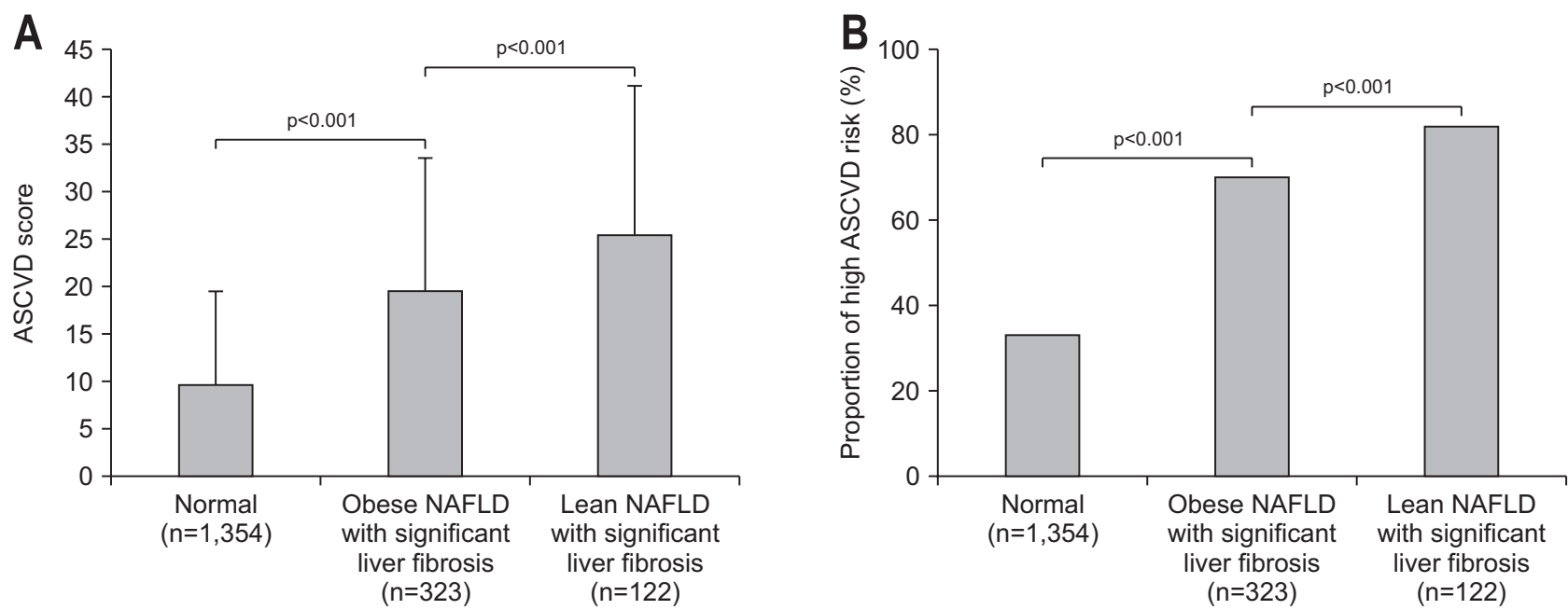

Fig. 3. ASCVD score and proportion of patients with high ASCVD risk according to NFS-defined significant liver fibrosis stratified by the CNSdefined NAFLD/obesity status. Lean subjects with NFS-defined significant liver fibrosis had a significantly higher ASCVD score (A) and prevalence of a high ASCVD risk (B) than obese subjects with significant liver fibrosis and those without significant liver fibrosis (all $p<0.001$ ).

ASCVD, atherosclerotic cardiovascular disease; NAFLD, nonalcoholic fatty liver disease; NFS, NAFLD fibrosis score; CNS, comprehensive NAFLD score.

Table 4. High Probability of ASCVD According to Obesity and Significant Fibrosis Based on the NAFLD Fibrosis Score

\begin{tabular}{|c|c|c|c|c|c|}
\hline \multirow{2}{*}{ Model } & \multirow{2}{*}{$\begin{array}{l}\text { NAFLD with no fibrosis } \\
\text { OR }(95 \% \mathrm{CI})\end{array}$} & \multicolumn{2}{|c|}{ Obese NAFLD subjects with significant fibrosis } & \multicolumn{2}{|c|}{ Lean NAFLD subjects with significant fibrosis } \\
\hline & & OR $(95 \% \mathrm{Cl})$ & $\mathrm{p}$-value & $\mathrm{OR}(95 \% \mathrm{Cl})$ & p-value \\
\hline Crude & 1.00 (reference) & $1.66(1.33-2.08)$ & $<0.001$ & $2.61(1.98-3.43)$ & $<0.001$ \\
\hline Model 1 & 1.00 (reference) & $2.20(1.59-3.06)$ & $<0.001$ & $2.53(1.40-4.56)$ & 0.002 \\
\hline Model 2 & 1.00 (reference) & $1.93(1.28-3.63)$ & 0.002 & $2.60(1.14-5.91)$ & 0.023 \\
\hline
\end{tabular}

ASCVD, atherosclerotic cardiovascular disease; NAFLD, nonalcoholic fatty liver disease; OR, odds ratio; $\mathrm{Cl}$, confidence interval.

Model 1: adjusted for age and sex and model 2: adjusted for age, sex, smoking, exercise, waist circumference, hypertension, diabetes, homeostasis model assessment of insulin resistance, chronic kidney disease, and hyper-low-density lipoprotein cholesterolemia.

ing NAFLD based on the LFS (Supplementary Table 3).

\section{Association between ASCVD risk and significant liver fibrosis}

Because fibrosis progression is significantly associated with an increased risk for ASCVD among subjects with NAFLD, ${ }^{32}$ we selected subjects with NAFLD for further statistical analyses $(n=1,799)$. Lean subjects with NFSdefined significant liver fibrosis had a significantly higher ASCVD score and prevalence of a high ASCVD risk (mean $25.6 \pm 15.7,82.0 \%$ ), followed by obese subjects with NFS- defined significant liver fibrosis and those without NFSdefined significant liver fibrosis (mean 19.5 $\pm 14.1,70.3 \%$; mean 9.7 \pm 9.8 , 33.3\%; respectively, all $\mathrm{p}<0.001$ ) (Fig. 3). When BARD was used to define significant liver fibrosis, similar findings were obtained (Supplementary Fig. 3).

\section{Association between a high probability of ASCVD and significant liver fibrosis}

The associations between a high probability of ASCVD and significant liver fibrosis stratified by obesity among subjects with NAFLD according to multistep adjustments 
are shown in Table 4. When NFS was used to define significant fibrosis and the relative risk of ASCVD was assessed after sufficient adjustment (model 2), subjects with lean NAFLD and significant liver fibrosis showed a significantly higher OR for the risk of ASCVD than subjects with obese NAFLD with or without significant liver fibrosis (odds ratio, 2.60 vs $1.93 ; \mathrm{p}=0.023$ ). When BARD was used to define significant liver fibrosis, similar findings were obtained (Supplementary Table 4).

\section{DISCUSSION}

A close association between NAFLD and obesity has consistently been reported. ${ }^{3,19}$ In this context, most subjects with NAFLD are overweight/obese and have varying components of metabolic syndrome. ${ }^{4,33}$ Nonetheless, a significant proportion of subjects with NAFLD are lean, although the clinical implications are unclear. ${ }^{34}$ Accordingly, using nationwide, population-based, cross-sectional data, we investigated whether ASCVD risk differs according to obesity in patients with NAFLD and checked whether similar results can be reproduced in subgroups with significant liver fibrosis.

Subjects with lean NAFLD had significantly higher ASCVD scores and a greater chance of having high ASCVD risk than those with obese NAFLD. Even if obesity was defined as $\mathrm{BMI} \geq 30 \mathrm{~kg} / \mathrm{m}^{2}$, our main findings were nearly the same. Hypertension, DM, hypo-HDL cholesterolemia, hypertriglyceridemia, and albuminuria significantly increased the risk of ASCVD in lean compared to obese subjects with NAFLD. After appropriate adjustment, lean subjects with NAFLD had a significantly higher ASCVD risk than obese subjects with or without NAFLD. Furthermore, after adjustment, lean subjects with significant liver fibrosis had the highest ASCVD risk, followed by obese subjects with significant liver fibrosis and those without significant liver fibrosis.

This study had several strengths. First, the selected cohort was large $(n>4,000)$, ensuring statistical reliability and robust results. Furthermore, the proportion of lean subjects with NAFLD in the cohort (11.0\%) was similar to that in previous Asian studies (9\% to $23.5 \%$ ). ${ }^{13,35}$ This suggests that the subjects were selected appropriately and that the results are applicable to other Asian populations, although further validation is required for other ethnic groups. In addition, the prevalence of subjects with NAFLD (37.6\%) was similar to that in a recent Asian study. ${ }^{36}$ Therefore, our study population, a nationwide representative cohort, was selected appropriately based on noninvasive surrogates for statistical analyses.
Second, to the best of our knowledge, this is the first report of an independent association between ASCVD risk and NAFLD according to obesity status. NAFLD and obesity are significant risk factors for cardiovascular events. ${ }^{17-19}$ However, after controlling for important confounders, lean NAFLD was associated with a higher ASCVD score and an increased prevalence of a high ASCVD risk compared to obese NAFLD; moreover, this finding was reproduced in the subgroup with significant liver fibrosis. In addition, our results provide evidence on the prognosis of lean NAFLD. Unlike the prognosis and complications of overall NAFLD, the data on lean NAFLD are scarce. Two studies have reported a higher mortality rate for lean compared to obese NAFLD. ${ }^{37,38}$ Moreover, CVD accounted for approximately one quarter of the causes of death. ${ }^{37}$ In our study, subjects with lean NAFLD were at markedly increased risk for individual cardiometabolic components and ASCVD compared to those with obese NAFLD. These data indicate that lean subjects with NAFLD should be counselled about the risk for unfavorable cardiovascular outcomes and managed accordingly. ${ }^{39}$

Third, the influence of NAFLD on ASCVD might have been biased because most subjects with NAFLD had simple hepatic steatosis, which has a very favorable prognosis. Thus, we selected subjects with NAFLD to determine whether the co-existence of significant liver fibrosis is significantly associated with ASCVD risk, because liver fibrosis can be considered a sequela of the inflammatory process of NAFLD. In addition, liver fibrosis is the single most important factor as well as a clinically relevant issue that correlates with poor outcomes. ${ }^{40}$ In the subgroup with NAFLD, around $20 \%$ of subjects had significant liver fibrosis, indicating that lean subjects had higher ASCVD risk than obese subjects among those with NAFLD and significant fibrosis.

We are also aware of several unresolved issues that should be addressed. First, although we used well-validated liver fibrosis and steatosis prediction models, liver imaging and histological information was not available because of the high cost of ultrasonography and ethical concerns regarding screening of a large national population-based cohort. KNHANES participants who gave informed consent underwent only serum tests. In addition, because cancer diagnosis in the KNHANES was based on a questionnaire, not ultrasonography, subjects with hepatocellular carcinoma were excluded.

Second, because of the cross-sectional nature of the study, we could not assess the longitudinal dynamic association between changes in NAFLD, obesity status, and ASCVD risk. We were also unable to assess the effects of therapeutic interventions, such as lifestyle modification, 
exercise, weight loss, medications, nutritional support, and protein supplements, on NAFLD, obesity, and ASCVD risk. Nevertheless, our results reveal the need to screen patients with NAFLD, particularly lean patients, to identify those at high risk for ASCVD requiring intensive medical therapy.

Third, we used a pooled cohort risk equation to assess ASCVD risk and did not examine the risk for real clinical events during follow-up. The 10-year ASCVD risk with primary prevention is estimated in the blood cholesterol guidelines of the ACC/AHA. However, because the equation for calculating ASCVD risk might have been in the study population, our findings should be interpreted with caution.

Finally, several serum markers, such as HbAlc, were available for only a small proportion of the subjects. Thus, the incremental influence on the final results, not simply the presence of DM, could not be assessed. In addition, detailed information regarding antihypertensive and antidiabetic drugs was not available, preventing analyses of their influence. Furthermore, although we excluded subjects known to have chronic liver diseases (such as viral hepatitis and alcoholic liver disease), those with other types of such diseases (such as Wilson disease, autoimmune liver disease, or primary biliary hepatopathy) might have been included, which may have biased the results. Moreover, as a result of the limitations of general medical examination, we could not adjust for dietary preference and genetic risk factors (including patatin-like phospholipase domaincontaining 3 and transmembrane 6 superfamily member 2 ), which could affect the risk for lean NAFLD. ${ }^{41}$

Although the characteristics and underlying pathophysiological mechanism of lean NAFLD/nonalcoholic steatohepatitis are unclear, individuals with a low BMI are likely to have reduced lean body mass, particularly muscle mass, which could lead to unfavorable traits related to cardiovascular outcomes. ${ }^{42}$ In addition, fat tissue in a distinct depot may have protective functions, particularly in people with chronic diseases. ${ }^{43}$ Subcutaneous fat can act as a metabolic reservoir, protecting other organs from lipotoxicity and ectopic fat formation, and leg fat is associated with lower ASCVD risk and cardiometabolic risk factors. ${ }^{44}$

In conclusion, this nationwide survey of a representative sample of Korean individuals demonstrated that, despite a more favorable metabolic profile, subjects with lean NAFLD had a significantly higher ASCVD score and prevalence of a high ASCVD risk than those with obese NAFLD. Similarly, lean subjects with significant liver fibrosis were at higher risk for ASCVD than obese subjects in the subpopulation with NAFLD. In this context, the presence of fatty liver should prompt clinicians to address

metabolic conditions that could modify the long-term outcomes, irrespective of body weight. Prospective, welldesigned, longitudinal studies are needed to elucidate the complex relationships among NAFLD, obesity, and cardiovascular risk.

\section{CONFLICTS OF INTEREST}

S.H.A. and S.U.K are editorial board members of the journal but were not involved in the peer reviewer selection, evaluation, or decision process of this article. No other potential conflicts of interest relevant to this article were reported.

\section{ACKNOWLEDGEMENTS}

This study was supported by Basic Science Research Program through the National Research Foundation of Korea (NRF) funded by the Ministry of Science, ICT and Future Planning (2019R1A2C4070136), by the Ministry of Education (2018R1D1A1B07050005), and by the Korea Healthcare Technology R\&D Project, Ministry of Health and Welfare (HI17C0913), Republic of Korea. The funders had no role in study design, data collection, and analysis, decision to publish, or preparation of the manuscript.

\section{AUTHOR CONTRIBUTIONS}

Conception and design: S.U.K., Y.H.L., Y.K., E.H. Development of methodology: S.U.K., Y.H.L., Y.K., E.H. Analysis and interpretation of data: S.U.K., Y.H.L., Y.K., E.H. Writing, review, and/or revision of the manuscript: S.U.K., Y.H.L., Y.K., E.H. Administrative, technical, or material support: S.U.K., Y.H.L., J.S.L., H.W.L., B.K.K., M.K.K., H.S.K., J.Y.P., D.Y.K., S.H.A., B.W.L., E.S.K., B.S.C., Y.K., E.H. Study supervision: S.U.K., Y.H.L.

\section{ORCID}

Yuna Kim Eugene Han Jae Seung Lee Hye Won Lee Beom Kyung Kim Mi Kyung Kim Hye Soon Kim Jun Yong Park https://orcid.org/0000-0003-1574-9121 https://orcid.org/0000-0002-3237-3317 https://orcid.org/0000-0002-2371-0967 https://orcid.org/0000-0002-3552-3560 https://orcid.org/0000-0002-5363-2496 https://orcid.org/0000-0002-7388-2660 https://orcid.org/0000-0001-6298-3506 https://orcid.org/0000-0001-6324-2224 
Do Young Kim

https://orcid.org/0000-0002-8327-3439

Sang Hoon Ahn

https://orcid.org/0000-0002-3629-4624

Byung-Wan Lee

Eun Seok Kang

Bong-Soo Cha

Yong-ho Lee

Seung Up Kim https://orcid.org/0000-0002-0364-4675

https://orcid.org/0000-0003-0542-2854

https://orcid.org/0000-0002-6219-4942

https://orcid.org/0000-0002-9658-8050

\section{SUPPLEMENTARY MATERIALS}

Supplementary materials can be accessed at https://doi. org/10.5009/gnl210084.

\section{REFERENCES}

1. Chalasani N, Younossi Z, Lavine JE, et al. The diagnosis and management of nonalcoholic fatty liver disease: practice guidance from the American Association for the Study of Liver Diseases. Hepatology 2018;67:328-357.

2. Younossi ZM, Koenig AB, Abdelatif D, Fazel Y, Henry L, Wymer M. Global epidemiology of nonalcoholic fatty liver disease: meta-analytic assessment of prevalence, incidence, and outcomes. Hepatology 2016;64:73-84.

3. Yoo JJ, Kim W, Kim MY, et al. Recent research trends and updates on nonalcoholic fatty liver disease. Clin Mol Hepatol 2019;25:1-11.

4. Rotundo L, Persaud A, Feurdean M, Ahlawat S, Kim HS. The association of leptin with severity of non-alcoholic fatty liver disease: a population-based study. Clin Mol Hepatol 2018;24:392-401.

5. Fargion S, Porzio M, Fracanzani AL. Nonalcoholic fatty liver disease and vascular disease: state-of-the-art. World J Gastroenterol 2014;20:13306-13324.

6. Ma J, Hwang SJ, Pedley A, et al. Bi-directional analysis between fatty liver and cardiovascular disease risk factors. J Hepatol 2017;66:390-397.

7. Golabi P, Fukui N, Paik J, Sayiner M, Mishra A, Younossi ZM. Mortality risk detected by atherosclerotic cardiovascular disease score in patients with nonalcoholic fatty liver disease. Hepatol Commun 2019;3:1050-1060.

8. Das K, Chowdhury A. Lean NASH: distinctiveness and clinical implication. Hepatol Int 2013;7 Suppl 2:806-813.

9. Fan JG, Kim SU, Wong VW. New trends on obesity and NAFLD in Asia. J Hepatol 2017;67:862-873.

10. Albhaisi S, Chowdhury A, Sanyal AJ. Non-alcoholic fatty liver disease in lean individuals. JHEP Rep 2019;1:329-341.

11. Sookoian S, Pirola CJ. Systematic review with meta-analysis: risk factors for non-alcoholic fatty liver disease suggest a shared altered metabolic and cardiovascular profile be-

tween lean and obese patients. Aliment Pharmacol Ther 2017;46:85-95.

12. Ando W, Yokomori H, Tsutsui N, et al. Serum matrix metalloproteinase-1 level represents disease activity as opposed to fibrosis in patients with histologically proven nonalcoholic steatohepatitis. Clin Mol Hepatol 2018;24:61-76.

13. Leung JC, Loong TC, Wei JL, et al. Histological severity and clinical outcomes of nonalcoholic fatty liver disease in nonobese patients. Hepatology 2017;65:54-64.

14. Younes R, Bugianesi E. NASH in lean individuals. Semin Liver Dis 2019;39:86-95.

15. Hagström H, Nasr P, Ekstedt M, et al. Cardiovascular risk factors in non-alcoholic fatty liver disease. Liver Int 2019;39:197-204.

16. Chen F, Esmaili S, Rogers GB, et al. Lean NAFLD: a distinct entity shaped by differential metabolic adaptation. Hepatology 2020;71:1213-1227.

17. Ahmed MH, Barakat S, Almobarak AO. Nonalcoholic fatty liver disease and cardiovascular disease: has the time come for cardiologists to be hepatologists? J Obes 2012;2012:483135.

18. Adams LA, Anstee QM, Tilg H, Targher G. Non-alcoholic fatty liver disease and its relationship with cardiovascular disease and other extrahepatic diseases. Gut 2017;66:11381153.

19. Polyzos SA, Kountouras J, Mantzoros CS. Obesity and nonalcoholic fatty liver disease: from pathophysiology to therapeutics. Metabolism 2019;92:82-97.

20. Kim Y. The Korea National Health and Nutrition Examination Survey (KNHANES): current status and challenges. Epidemiol Health 2014;36:e2014002.

21. Lee YH, Bang H, Park YM, et al. Non-laboratory-based self-assessment screening score for non-alcoholic fatty liver disease: development, validation and comparison with other scores. PLoS One 2014;9:e107584.

22. Kotronen A, Peltonen M, Hakkarainen A, et al. Prediction of non-alcoholic fatty liver disease and liver fat using metabolic and genetic factors. Gastroenterology 2009;137:865-872.

23. Cichoż-Lach H, Celiński K, Prozorow-Król B, Swatek J, Słomka M, Lach T. The BARD score and the NAFLD fibrosis score in the assessment of advanced liver fibrosis in nonalcoholic fatty liver disease. Med Sci Monit 2012;18:CR735CR740.

24. Goff DC Jr, Lloyd-Jones DM, Bennett G, et al. 2013 ACC/ AHA guideline on the assessment of cardiovascular risk: a report of the American College of Cardiology/American Heart Association Task Force on Practice Guidelines. Circulation 2014;129(25 Suppl 2):S49-S73.

25. Oh SW. Obesity and metabolic syndrome in Korea. Diabetes Metab J 2011;35:561-566.

26. Levey AS, Stevens LA, Schmid CH, et al. A new equation 
to estimate glomerular filtration rate. Ann Intern Med 2009;150:604-612.

27. National Kidney Foundation. K/DOQI clinical practice guidelines for chronic kidney disease: evaluation, classification, and stratification. Am J Kidney Dis 2002;39(2 Suppl 1):S1-S266.

28. Grundy SM, Cleeman JI, Merz CN, et al. Implications of recent clinical trials for the National Cholesterol Education Program Adult Treatment Panel III guidelines. Circulation 2004;110:227-239.

29. Lee YH, Kim SU, Song K, et al. Sarcopenia is associated with significant liver fibrosis independently of obesity and insulin resistance in nonalcoholic fatty liver disease: nationwide surveys (KNHANES 2008-2011). Hepatology 2016;63:776-786.

30. Lee YH, Kim JE, Roh YH, et al. The combination of vitamin $\mathrm{D}$ deficiency and mild to moderate chronic kidney disease is associated with low bone mineral density and deteriorated femoral microarchitecture: results from the KNHANES 2008-2011. J Clin Endocrinol Metab 2014;99:3879-3888.

31. Matthews DR, Hosker JP, Rudenski AS, Naylor BA, Treacher DF, Turner RC. Homeostasis model assessment: insulin resistance and beta-cell function from fasting plasma glucose and insulin concentrations in man. Diabetologia 1985;28:412-419.

32. Targher G, Byrne CD, Lonardo A, Zoppini G, Barbui C. Non-alcoholic fatty liver disease and risk of incident cardiovascular disease: a meta-analysis. J Hepatol 2016;65:589-600.

33. Milić S, Lulić D, Štimac D. Non-alcoholic fatty liver disease and obesity: biochemical, metabolic and clinical presentations. World J Gastroenterol 2014;20:9330-9337.

34. Curcic IB, Berkovic MC, Kuna L, et al. Obesity paradox in chronic liver diseases: product of bias or a real thing? J Clin Transl Hepatol 2019;7:275-279.

35. Kwon YM, Oh SW, Hwang SS, Lee C, Kwon H, Chung GE. Association of nonalcoholic fatty liver disease with compo- nents of metabolic syndrome according to body mass index in Korean adults. Am J Gastroenterol 2012;107:1852-1858.

36. Younossi Z, Anstee QM, Marietti M, et al. Global burden of NAFLD and NASH: trends, predictions, risk factors and prevention. Nat Rev Gastroenterol Hepatol 2018;15:11-20.

37. Zou B, Yeo YH, Nguyen VH, Cheung R, Ingelsson E, Nguyen $\mathrm{MH}$. Prevalence, characteristics and mortality outcomes of obese, nonobese and lean NAFLD in the United States, 1999-2016. J Intern Med 2020;288:139-151.

38. Golabi P, Paik JM, Arshad T, Younossi Y, Mishra A, Younossi ZM. Mortality of NAFLD according to the body composition and presence of metabolic abnormalities. Hepatol Commun 2020;4:1136-1148.

39. Golabi P, Paik J, Fukui N, Locklear CT, de Avilla L, Younossi ZM. Patients with lean nonalcoholic fatty liver disease are metabolically abnormal and have a higher risk for mortality. Clin Diabetes 2019;37:65-72.

40. Ekstedt M, Hagström H, Nasr P, et al. Fibrosis stage is the strongest predictor for disease-specific mortality in NAFLD after up to 33 years of follow-up. Hepatology 2015;61:15471554.

41. Kim D, Kim WR. Nonobese fatty liver disease. Clin Gastroenterol Hepatol 2017;15:474-485.

42. Han E, Lee YH, Kim YD, et al. Nonalcoholic fatty liver disease and sarcopenia are independently associated with cardiovascular risk. Am J Gastroenterol 2020;115:584-595.

43. Lavie CJ, De Schutter A, Patel DA, Romero-Corral A, Artham SM, Milani RV. Body composition and survival in stable coronary heart disease: impact of lean mass index and body fat in the "obesity paradox". J Am Coll Cardiol 2012;60:1374-1380.

44. Han E, Lee YH, Lee BW, Kang ES, Lee IK, Cha BS. Anatomic fat depots and cardiovascular risk: a focus on the leg fat using nationwide surveys (KNHANES 2008-2011). Cardiovasc Diabetol 2017;16:54. 